\title{
Discovery of Black Dye Crystal Structure Polymorphs: Implications for Dye Conformational Variation in Dye- Sensitized Solar Cells
}

Jacqueline M. Cole, ${ }^{*,+\ddagger}$ Kian Sing Low ${ }^{\dagger}$, Yun Gong ${ }^{\dagger}$

${ }^{\dagger}$ Cavendish Laboratory, University of Cambridge, J. J. Thomson Avenue, Cambridge, CB3 oHE, U.K.

${ }^{\ddagger}$ Argonne National Laboratory, 9700 S Cass Avenue, Argonne, IL 60439, USA

*Author for correspondence E-mail: jmc61@cam.ac.uk

SUPPORTING INFORMATION

Bond Geometry survey of NCS groups in all published ruthenium-based crystal structures via the Cambridge Structural Database

Table of Contents:

(a) Statistical distribution of N-C bond lengths in Ru-coordinated NCS groups

pS-2

(b) Statistical distribution of C-S bond lengths in Ru-coordinated NCS groups

pS-3

(c) Statistical distribution of NCS bond angles in Ru-coordinated NCS groups

pS-4 
(a) Statistical distribution of N-C bond lengths in Ru-coordinated NCS groups

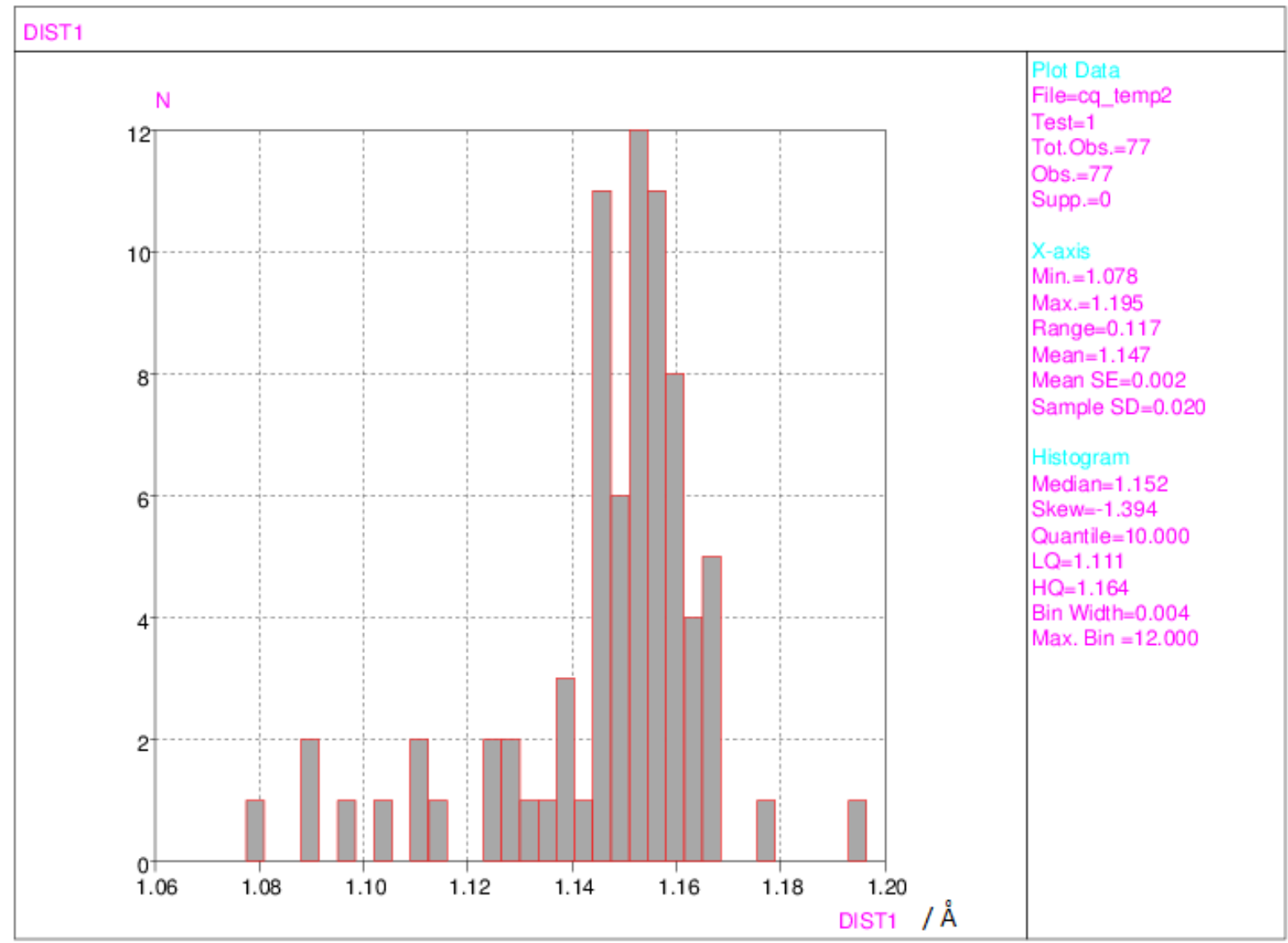

Figure S1(a): Histogram of frequency distribution for $\mathrm{N}-\mathrm{C}$ bond lengths contained in the Cambridge Structural Database in molecules that feature Ru-coordinated NCS groups. 
(b) Statistical distribution of C-S bond lengths in Ru-coordinated NCS groups

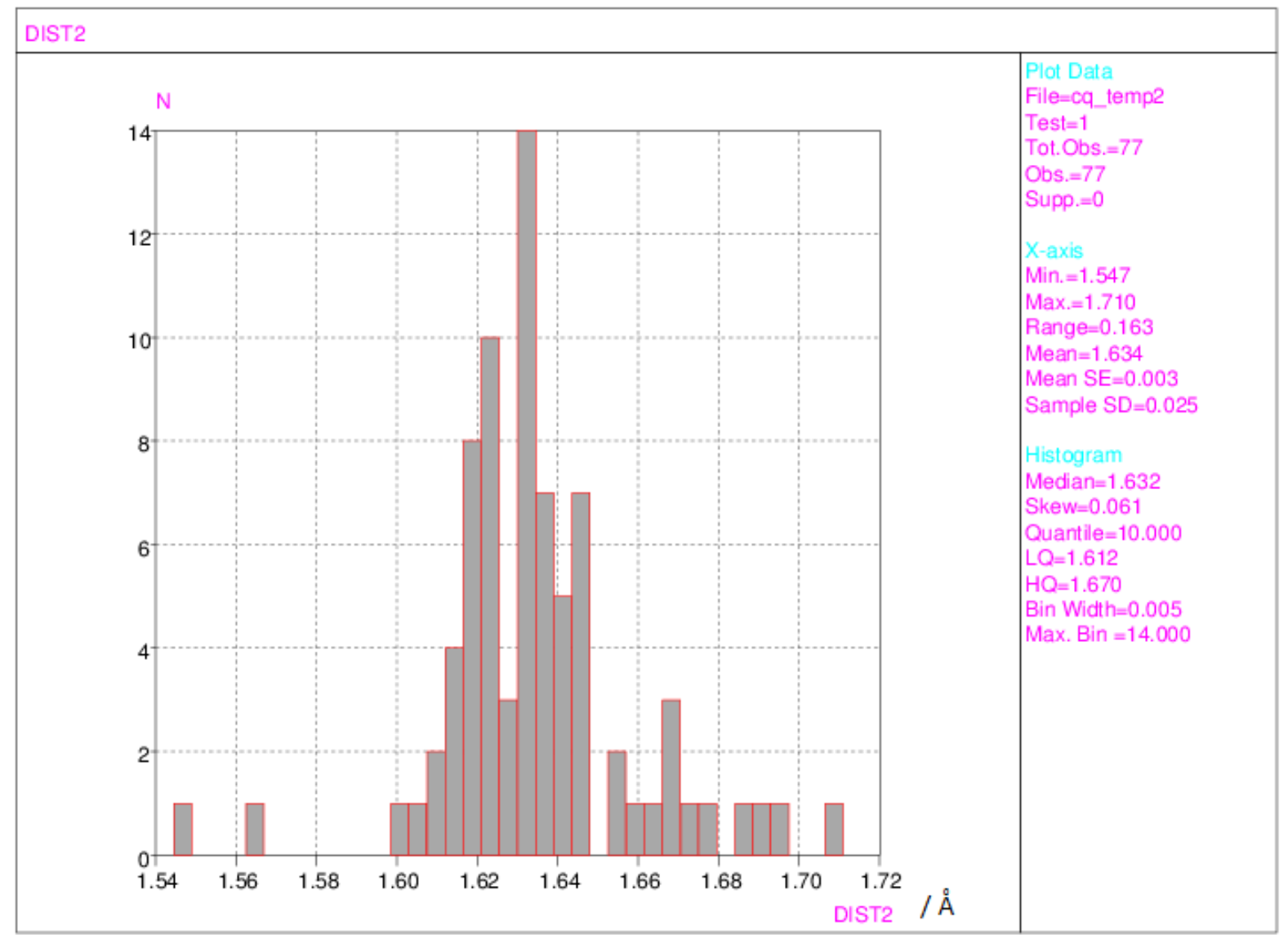

Figure S1(b): Histogram of frequency distribution for C-S bond lengths contained in the Cambridge Structural Database in molecules that feature Ru-coordinated NCS groups. 
(c) Statistical distribution of NCS bond angles in Ru-coordinated NCS groups

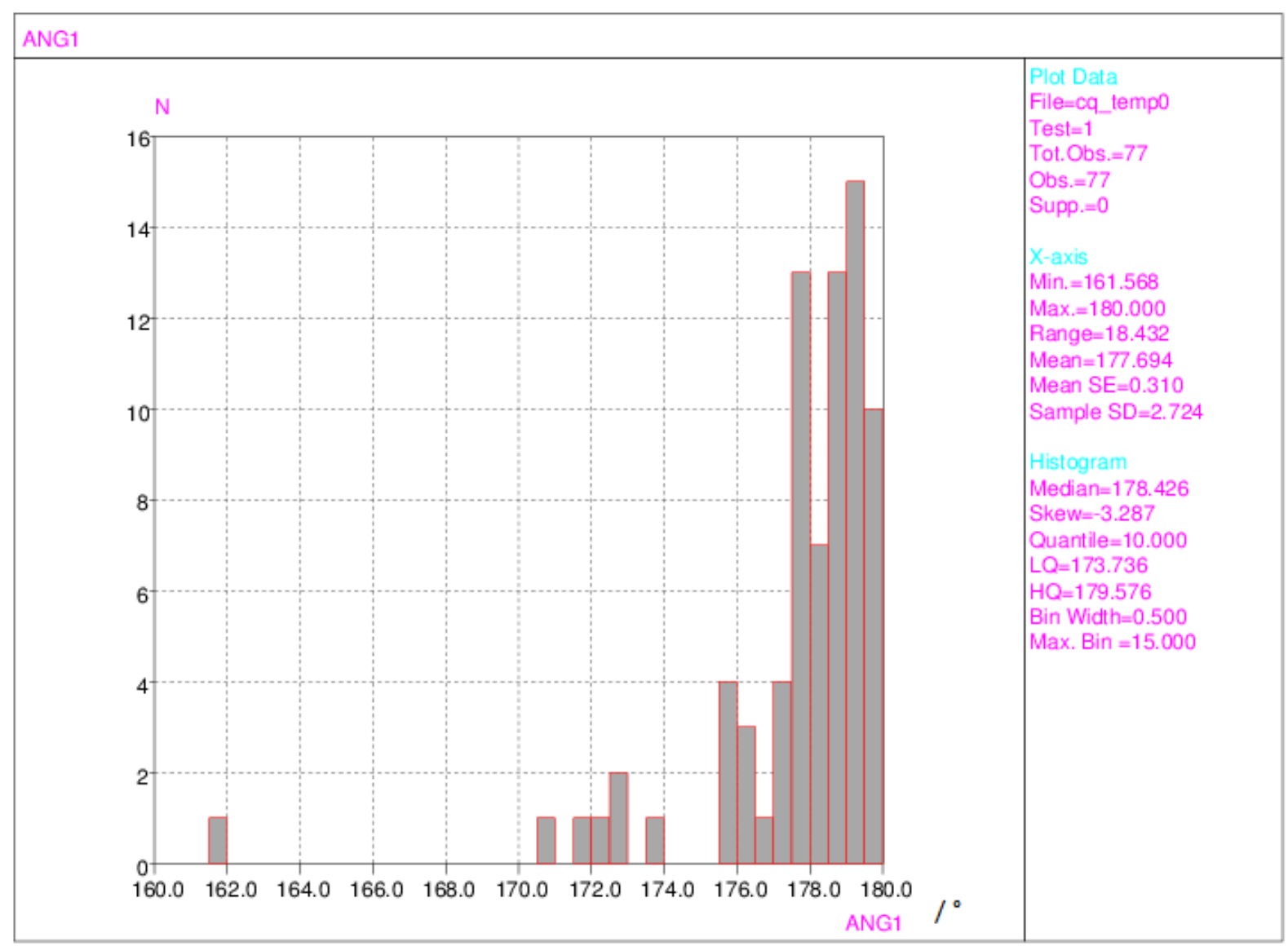

Figure S1(c): Histogram of frequency distribution for NCS bond angles contained in the Cambridge Structural Database in molecules that feature Ru-coordinated NCS groups. 